\title{
Hydrodynamic Analysis of AUV Hulls Using Semi-empirical and CFD Approach
}

\author{
Madan A. D*, Manoj T. Issac \\ Department of Ship Technology, Cochin University of Science and Technology, India
}

Copyright $\mathrm{O} 2017$ by authors, all rights reserved. Authors agree that this article remains permanently open access under the terms of the Creative Commons Attribution License 4.0 International License

\begin{abstract}
The prediction of hydrodynamic performance of AUV is essential during the early stages of design for the efficient modeling of the AUV. This paper discusses the use of semi-empirical equations developed for airship hulls by various researchers in the field of aerodynamics, on the axisymmetric AUV bare hull at different angles of attack. Three hulls of varying length-to-diameter (L/D) ratios are selected for the study. The results obtained from the semi-empirical equations are compared with the available experimental results and also with the results obtained from the numerical analysis carried out using the CFD code - STAR CCM+.
\end{abstract}

Keywords AUV (Autonomous Underwater Vehicles), Hydrodynamic Forces and Moments, Semi-empirical Equations, CFD, STAR CCM+

\section{Introduction}

For many years, the ocean was being explored from the ships on its surfaces. To explore the ocean under the surface, evolved innovative idea of underwater vehicles. Underwater vehicles are capable of performing a pre-programmed mission while being submerged. The demand to make the vehicles less expensive and lightweight and to minimize the human error developed the unmanned underwater vehicles which can operate and navigate without a human administrator.

AUV is a robotic unmanned vehicle which can perform long range missions. It can be manoeuvred in 3 dimensional space and are controlled and guided by sensors and various on board systems which are pre-programmed. Depending on their mission requirements, AUVs have various shape and size. They can be categorized as - Survey class AUVs and Work Class AUVs. Survey class AUVs have streamlined body which experiences low drag, as it maintains the flow around the hull to be laminar for most of its length. These are assigned to carry out missions which last for several hours to several days and the power supply on board is limited. It is thus essential for a survey class AUV to make efficient use of power. A typical survey class torpedo-shaped AUV hull is shown in figure 1.

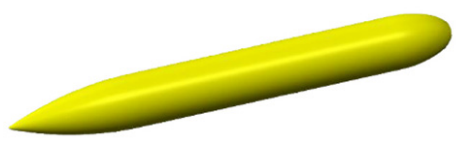

Figure 1. Survey class Torpedo - shaped AUV hull

\section{Hydrodynamic Bare Hull Forces and Coefficients}

An AUV hull submerged in water moving at an angle of attack experiences a force at a point called the centre of pressure. The force has two components - a parallel component or the Drag force which is in the direction of flow and a component perpendicular to the flow or the Lift force. Additionally, a component of this force also develops a torque along the Pitching axis of the hull. This is known as the Pitching moment. Hydrodynamic coefficients are dimensionless quantities which relate the hydrodynamic forces and moment on the body to the fluid density, flow velocity and a reference area. It is noted that the reference area is fixed throughout the procedure of calculation of the hydrodynamic parameters. The reference area can be the wetted surface area or the cross - sectional area or ' $L^{2}$ ' or ' $V^{2 / 3}$ ' where $L$ and $V$ are the length and volume of the AUV hull.

Methods to determine the hydrodynamic coefficients can broadly be classified as Test based methods and Predictive methods. Test based methods include captive model tests in a wind tunnel or a tow tank, free running model tests, etc. Usually, the hydrodynamic parameters of an AUV hull are determined at early stages of design. Major drawback of the test based methods is the requirement of a physical model, a laboratory and testing facilities which makes this method not very cost efficient. When cost is a major restriction, one can opt for the predictive method as an alternative which can be 
incorporated during the design phase of the vehicle and they are likely to yield satisfactory results. These are the CFD (Computational Fluid dynamics) Techniques and the semi-empirical techniques. The Semi-empirical approach is easier to apply, can be computed fast and are likely to yield satisfactory results. Semi-empirical equations proposed by various researchers to study the aerodynamic characteristics of axisymmetric airship hulls are discussed in this paper and later these equations are applied to predict the hydrodynamic characteristics of an axisymmetric AUV bare hull.

The investigation was started by Munk [1] who first proposed the method for calculating the hull forces on a rigid airship hull using the potential flow considerations only. The equations proposed by Munk for the resultant moment and normal force per unit length are:

$$
\begin{array}{r}
M=\left(k_{2}-k_{1}\right) \frac{\rho V^{2}}{2} \sin (2 \alpha) \\
f_{N}=\frac{d F}{d x}=\left(k_{2}-k_{1}\right) \frac{\rho V^{2}}{2} \sin (2 \alpha) \frac{d S}{d x}
\end{array}
$$

Here, $\left(k_{2}-k_{1}\right)$ is the apparent mass factor as given in Munk [1], $\rho$ is the density of water, $V$ is the free stream velocity, $F$ is the total normal force, $S$ is the cross sectional area, $x$ is the longitudinal distance along the hull from the nose and $\alpha$ is the angle of attack.

Allen and Perkins [2], [3], [4] continued Munk's work and declared that the Munk's formula was only valid for inviscid flows. They developed the following set of equations for lift, drag and pitching moment coefficients considering the effects of viscosity with the potential flow considerations.

$$
\begin{gathered}
C_{L}=2\left(k_{2}-k_{1}\right) \frac{S_{b}}{A} \alpha+\eta C_{D C} \frac{A_{p}}{A} \alpha^{2} \\
\Delta C_{D F}=C_{D F}-C_{D F(\alpha=0)}=\left(k_{2}-k_{1}\right) \frac{S_{b}}{A} \alpha^{2}+\eta C_{D C} \frac{A_{p}}{A} \alpha^{3} \\
C_{M}=2\left(k_{2}-k_{1}\right)\left(\frac{V_{B}-S_{b}\left(1-x_{m}\right)}{A X}\right) \alpha+\eta C_{D C} \frac{A_{p}}{A}\left(\frac{x_{m}-x_{p}}{X}\right) \alpha^{2}
\end{gathered}
$$

Here, $\eta$ is the cross flow drag proportionality factor, $C_{D C}$ is the cross flow drag coefficient, $S_{b}$ is the cross sectional area of the base, $A$ is the reference area, $A_{p}$ is the planform area of the hull, $V_{b}$ is the volume of the hull, $X$ is the reference length, $x_{p}$ is the distance from the nose to the centroid of the planform area, $\mathrm{xm}$ is the distance from the nose to the pitching moment center, $\Delta C_{D F}$ is the incremental drag force coefficient and $C_{D F}(\alpha=0)$ is the drag force coefficient at zero angle of attack. The first term on the right hand side of the equations (3), (4) and (5), represent the contributions of potential flow and the second term represent the contribution of the viscous flow.

Later, Hopkins [5] stated that the potential flow would govern only the forward portion of the hull up to a certain length $x_{o}$ and beyond which viscosity would govern the flow.

$$
\begin{gathered}
C_{L}=\frac{2 \alpha\left(k_{2}-k_{1}\right)}{V_{B}^{2 / 3}} \int_{0}^{x} \frac{d S}{d x} d x+\frac{2 \alpha^{2}}{V_{B}^{2 / 3}} \int_{x}^{L} \eta R_{x} C_{D C} d x \\
\Delta C_{D}=\frac{2 \alpha^{2}\left(k_{2}-k_{1}\right)}{V_{B}^{2 / 3}} \int_{0}^{x} \frac{d S}{d x} d x+\frac{2 \alpha^{3}}{V_{B}^{2 / 3}} \int_{x_{v}}^{L} \eta R_{x} C_{D C} d x \\
C_{M}=\frac{2 \alpha\left(k_{2}-k_{1}\right)}{V_{B}} \int_{0}^{x_{0}} \frac{d S}{d x}\left(x_{m}-x\right) d x+\frac{2 \alpha^{2}}{V_{B}} \int_{x_{x}}^{L} \eta R_{x} C_{D C}\left(x_{m}-x\right) d x
\end{gathered}
$$

$R_{x}$ is the radius of the hull at station $x$. In the equations (6), (7) and (8), the potential flow is considered up to a longitudinal distance $x_{o}$, hence integrated from 0 to $x_{o}$ and the viscous flow is considered from $x_{o}$ to the remaining length, $L$.

$x_{o}$ is determined by an empirically derived expression as mentioned in Hopkins [5].

$$
\frac{x_{o}}{l}=0.378+0.527 \frac{x_{1}}{l}
$$

where, $x_{I}$ is the longitudinal distance from the nose at which $\frac{d S}{d x}$ has a maximum negative value.

Based on the work of Allen and Perkins and Hopkins, Jorgensen[6] presented a method for computing axial force, normal force and pitching moment coefficient for large range of angles of attack (0 -180) mainly for the space shuttle like bodies. According to Evans [7], to apply the formulations for the underwater bodies, $\left(k_{2}-k_{l}\right)$ has to be included in the equations, which was neglected in the original Jorgensen's equations. Following are the modified equations for normal force, axial force and pitching moment coefficients:

For $0^{\circ} \leq \alpha \leq 180^{\circ}$

$$
C_{N}=\frac{S_{b}}{A}\left(k_{2}-k_{1}\right) \sin 2 \alpha^{\prime} \cos \frac{\alpha^{\prime}}{2}+\eta C_{D C} \frac{A_{p}}{A} \sin ^{2} \alpha^{\prime}
$$

$$
\begin{gathered}
\text { For } 0^{\circ} \leq \alpha \leq 90^{\circ} \\
C_{A}=C_{D F(\alpha=0)} \cos ^{2} \alpha^{\prime} \\
C_{M}=\left(k_{2}-k_{1}\right)\left(\frac{V_{B}-S_{b}\left(1-x_{m}\right)}{A d}\right) \sin 2 \alpha^{\prime} \cos \frac{\alpha^{\prime}}{2}+\eta C_{D C} \frac{A_{p}}{A}\left(\frac{x_{m}-x_{p}}{d}\right) \sin ^{2} \alpha^{\prime} \\
\text { For } 90^{\circ} \leq \alpha \leq 180^{\circ} \\
C_{A}=C_{D F(\alpha=180)} \cos ^{2} \alpha^{\prime} \\
C_{M}=\left(\frac{V_{B}-S_{b} x_{m}}{A d}\right) \sin 2 \alpha^{\prime} \cos \frac{\alpha^{\prime}}{2}+\eta C_{D C} \frac{A_{p}}{A}\left(\frac{x_{m}-x_{p}}{d}\right) \sin ^{2} \alpha^{\prime}
\end{gathered}
$$


Here, $\alpha^{\prime}=\alpha$ for $0^{\circ} \leq \alpha \leq 90^{\circ}$ and $\alpha^{\prime}=180-\alpha$ for $90^{\circ} \leq \alpha \leq 180^{\circ}$.

Nahon [8], [9], was the first researcher to model his ARCS AUV using the airship theories mentioned above. Similar work was carried out by, Prestro [10], Perrault [11], Evans [7], Barros et al. [12] etc.. The range of angle of attack up to which these equations can predict the hydrodynamic forces and moments with reasonable accuracy on the AUV hull is still unknown. Being a cost effective approach that require only the description of geometrical parameters of the vehicle, this method can be preferred over experimental and numerical methods at early stages of design.

\section{Modelling the Bare Hull}

Williams et al. [13] conducted tow tank tests on AUV bare - hull models of five different $\mathrm{L} / \mathrm{D}$ ratios at a constant speed of $2 \mathrm{~m} / \mathrm{s}$. Three of the five L/D (Length to Diameter) ratio hulls $(8.5,10.5$ and 12.5$)$ were chosen for this study, the particulars of which are listed in the Table 1 and the schematics are shown in the figure 2 . The experimental results are taken from the work of Williams et al. [13]

Table 1. Particulars of the three models used for the study (Williams et al. [13])

\begin{tabular}{|c|c|c|c|}
\hline $\begin{array}{c}\text { L/D } \\
\text { Ratio }\end{array}$ & LOA $(\mathrm{mm})$ & Nose Length $(\mathrm{mm})$ & Tail length $(\mathrm{mm})$ \\
\hline 8.5 & 1724 & 220 & 494 \\
\hline 10.5 & 2130 & 220 & 494 \\
\hline 12.5 & 2536 & 220 & 494 \\
\hline
\end{tabular}
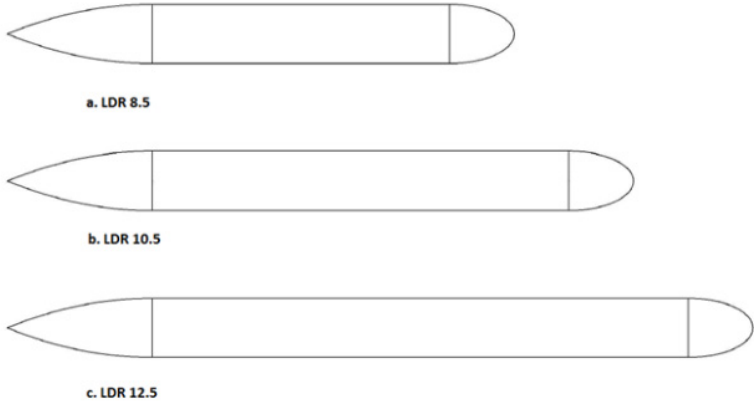

Figure 2. Schematic of the three models used for the study (a) L/D ratio 8.5 (b) L/D ratio 10.5 (c) L/D ratio 12.5

\section{Hydrodynamic Forces and Moments on the AUV Bare Hull (Using Semi-empirical Equations)}

\subsection{Lift Force for L/D Ratios $-8.5,10.5$ and 12.5}

The lift force increases with increase in angle of attack for each of the three L/D ratio hulls as shown in Figure 3.
The results from the semi-empirical equations predicted by Allen \& Perkins and Hopkins match well with the experimental results whereas Jorgensen's equations seems to agree well with the experimental results up to around 10 degrees angle of attack and beyond which the error is observed to increase. In general, the semi-empirical equations seem to predict the lift force with reasonable accuracy up to angles of attack of 10 degrees, beyond which the error in prediction increases with increasing angle of attack.

\section{L/D ratio 8.5}

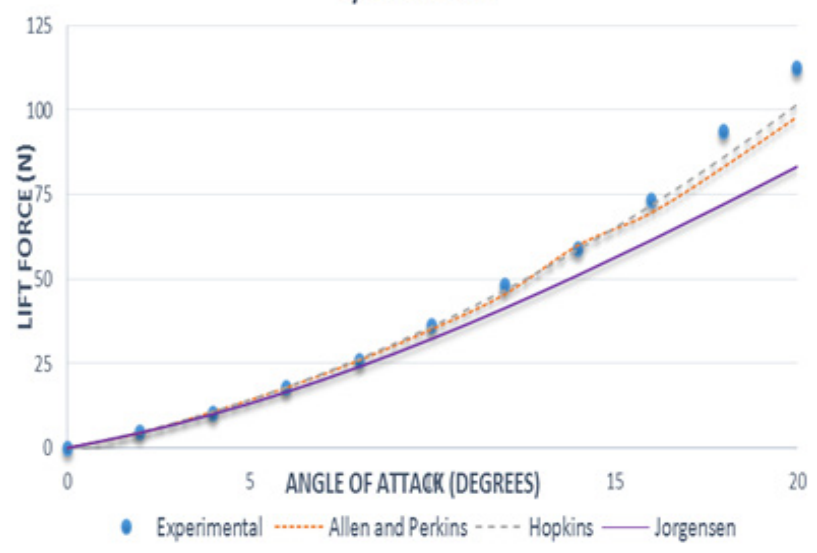

(a)

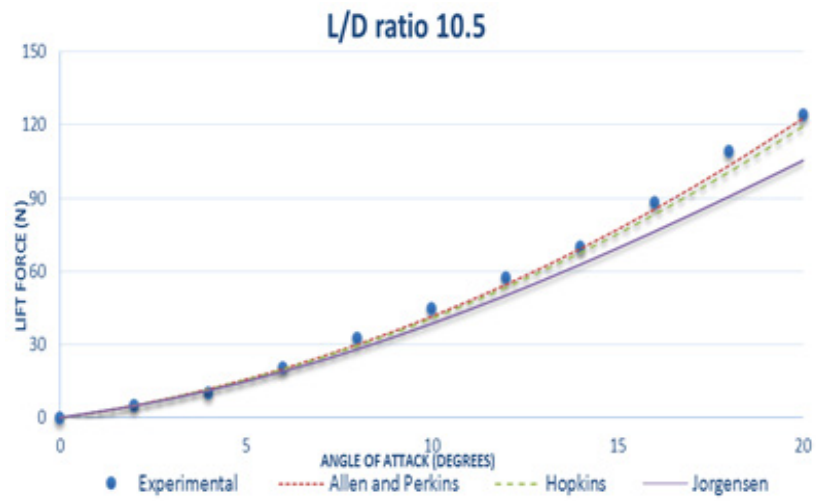

(b)

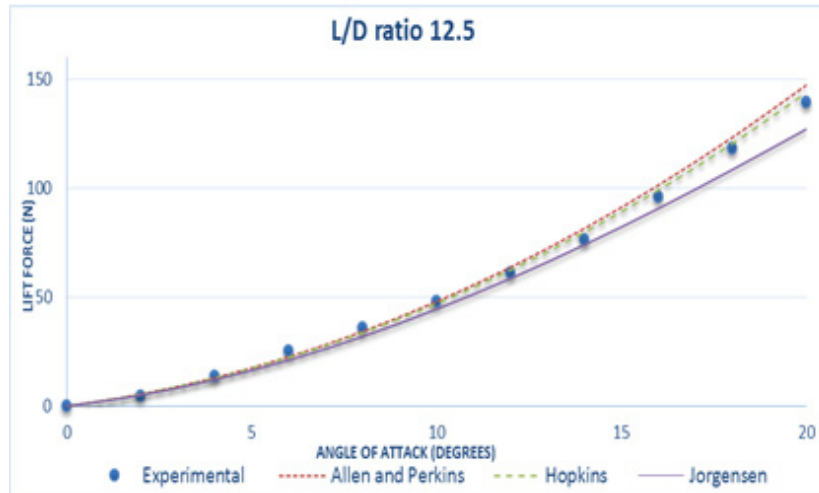

(c)

Figure 3. Lift force using various semi-empirical equations for $\mathrm{L} / \mathrm{D}$ ratios (a) 8.5 (b) 10.5 (c) 12.5 


\subsection{Drag Force for $L / D$ ratios-8.5, 10.5 and 12.5}

The minium drag force calculated using the semi-empirical methods seem to agree well with the minimum drag force (i.e. at zero degree angle of attack) from experiments reported in Williams et al. [13]. Figure 4 shows that for small angles of attack up to 10 degrees, the semi-empirical equations yield satisfactory results. It is observed that at higher angles of attack (beyond 10 degrees), the drag force is being over predicted by the Allen and Perkins' and Jorgensen's equations while Hopkin's formulation tends to agree reasonably well with th experimental results. Note that in the case of lift force, the predictions by both Allen \& Perkins and Hopkin's formulations agreed well with the experimental results.

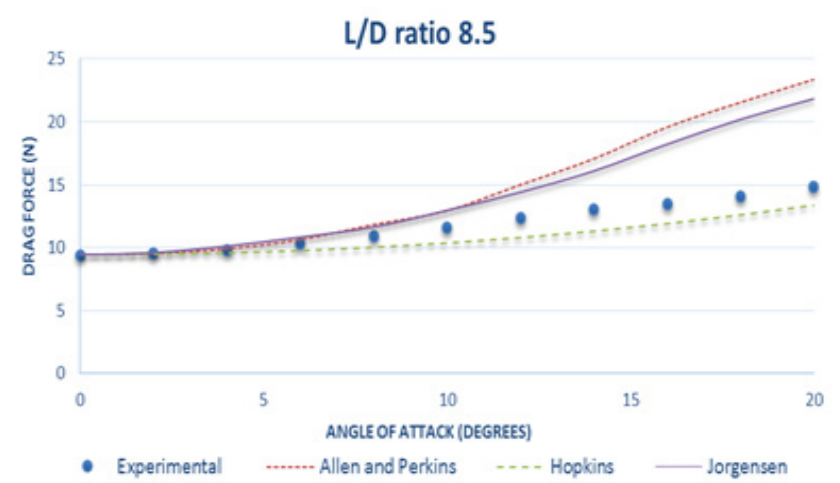

(a)

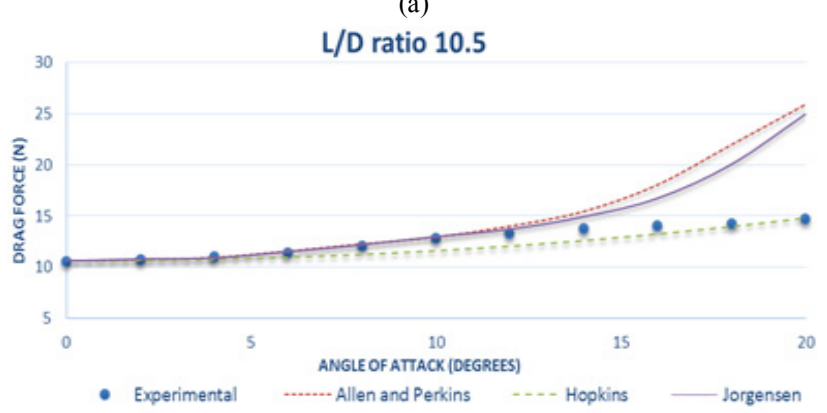

(b)

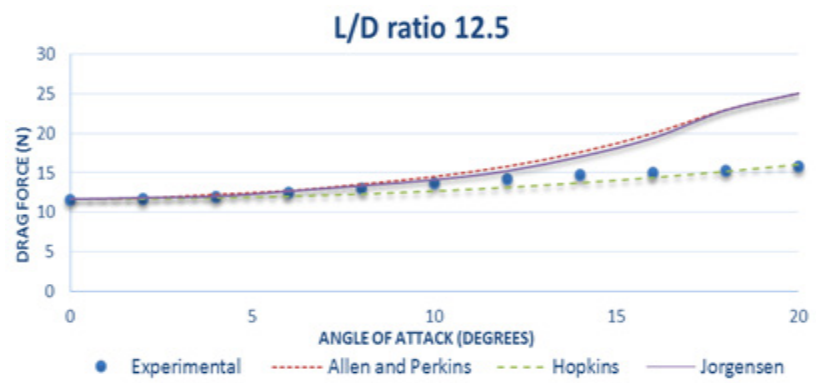

(c)

Figure 4. Drag force using various semi-empirical equations for $\mathrm{L} / \mathrm{D}$ ratio (a) 8.5 (b) 10.5 (c) 12.5

From figures 3 and 4 it can be inferred that maximum errors in the prediction of lift and drag forces were observed from Jorgensen's equations while Hopkin's equations are best suited for predicting both lift and drag forces

\subsection{Pitching Moment for L/D Ratios-8.5, 10.5 and 12.5}

Errors for pitching moment at higher angles of attack is found to be larger when compared with the errors of Lift and Drag forces. As in the case of Lift and Drag forces, semi-empirical equations have predicted the pitching moment reasonably well for small range of angles of attack of up to 5 degrees after which the errors increase as shown in the Figure 5.

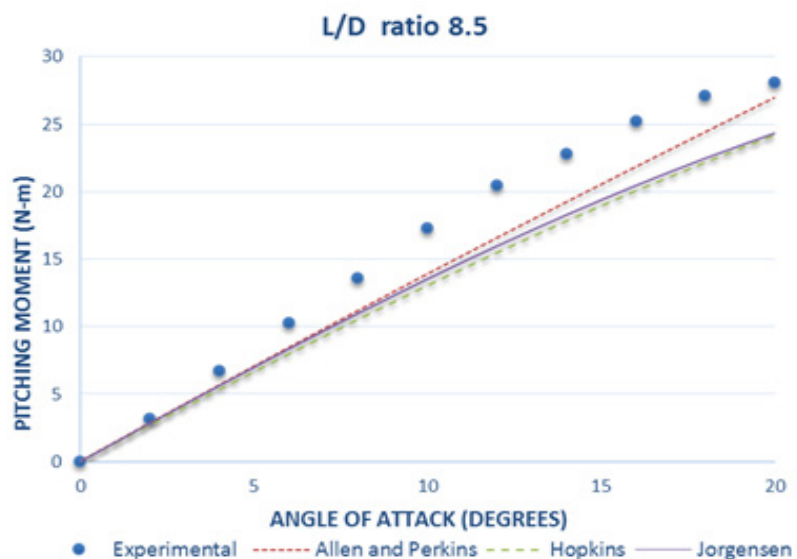

(a)

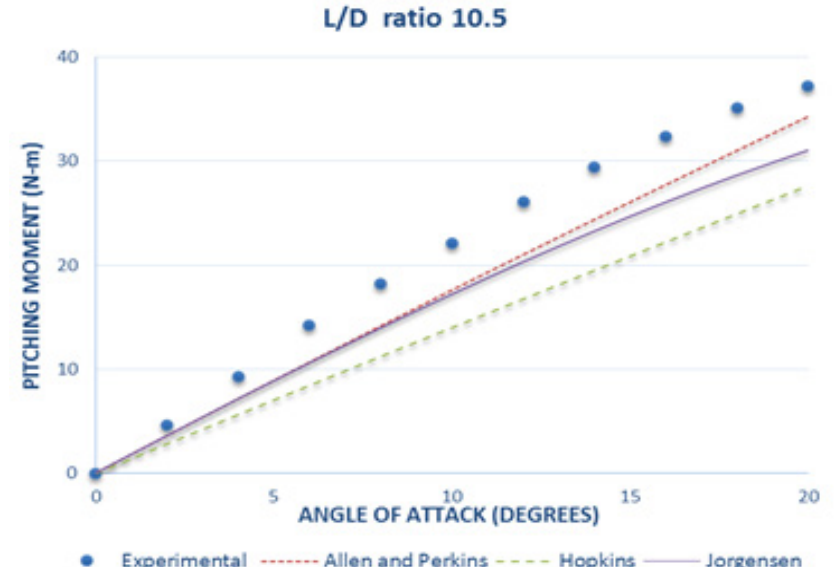

(b)

L/D ratio 12.5

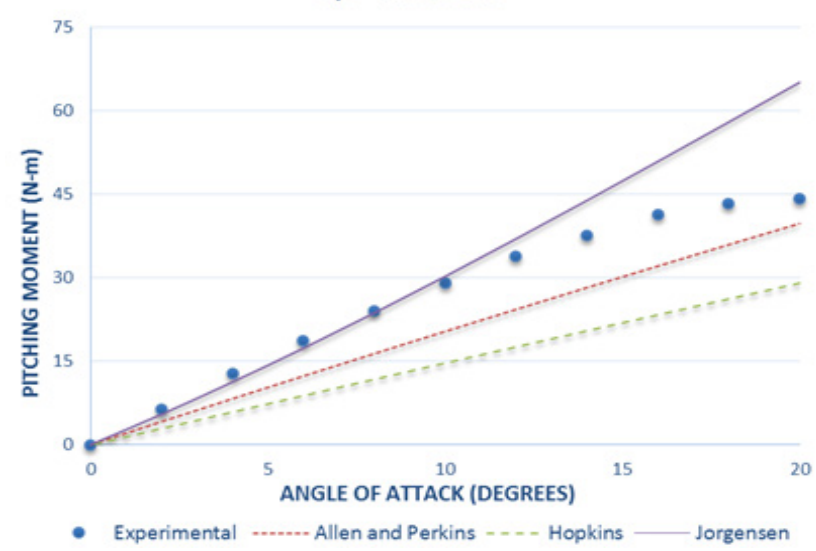

(c)

Figure 5. Pitching moment using various semi-empirical equations for L/D ratio (a) 8.5 (b) 10.5 (c) 12.5 
Observing the plots obtained from the semi-empirical equations by Allen and Perkins, Hopkins and Jorgensen, the predictions by the Hopkins equation is seen to be better than the other two, except for the pitching moment. The reason for Hopkins equations' results to be better than the other two (for lift and drag force) can be justified by the fact that the Hopkins equations solves for the potential flow and viscous flow separately over only the respective portions of the hull where they dominate.

\section{Hydrodynamic Forces and Moments on the AUV Bare Hull (using CFD)}

Before moving into the numerical approach of CFD, it is advisable to know the overview of the flow around the AUV. When the AUV hull moves around through the water, it displaces water. This in turn constructs complex flow patterns around the hull which is difficult to study. Here, the simulations are conducted at a Reynolds number in the order of $10^{5}$. Hence, the hull is surrounded by turbulent boundary layer. Due to the axisymmetric and slender shape of the hull, boundary layer flow separation is prevented until it reaches the aft body.

\subsection{Pre - Processing}

In this study, to solve the problem numerically, a commercial CFD tool STAR CCM+ (Simulation of turbulent flow in arbitrary regions Computational continuum mechanics + ) was used. The flow domain for the study was selected by using the base domain size as used by Jagadeesh et al. [14] and a domain independence analysis was carried out with other sizes to arrive at a flow domain for the study as shown in the Figure 6 (a). It is of the dimension 40D x 10D x 10D, where $\mathrm{D}$ is the maximum diameter of the AUV hull. The hull was placed near the inlet of the domain, so that more regions are given for the flow to get fully developed at the aft region.

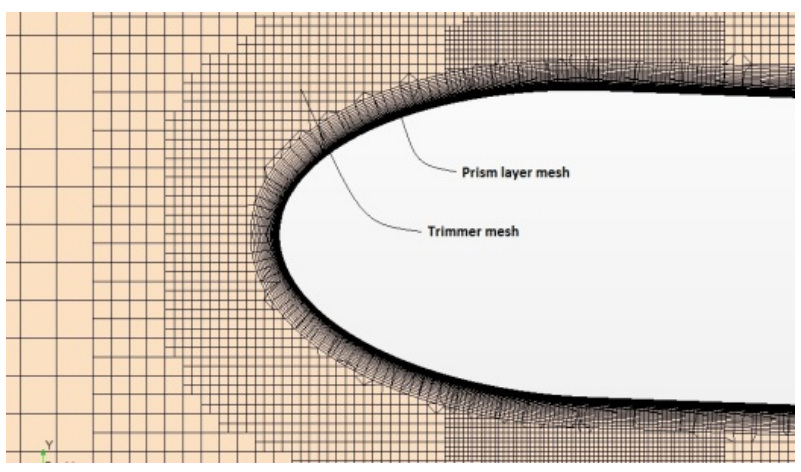

(a)

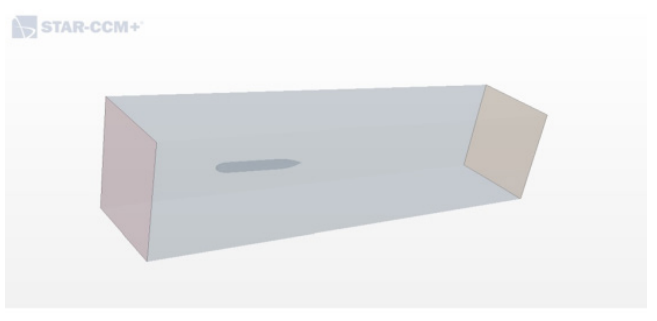

(a)

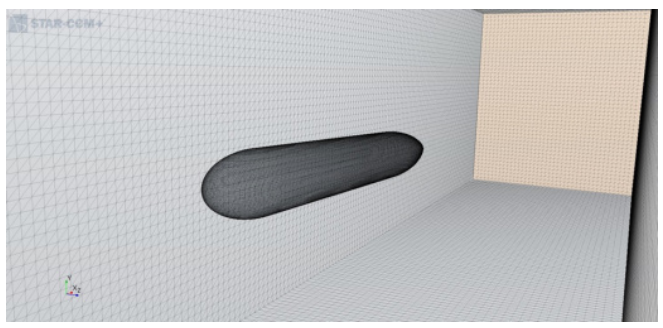

(b)

Figure 6. (a) Flow domain used for the numerical simulation (b) Typical volume mesh used for the numerical simulation

\subsubsection{Mesh generation and Physics model}

STAR CCM+ has a powerful semi-automatic mesh generator which generates both surface mesh and volume mesh based on the inputs given by the user. This study used the trimmer mesh as shown in the Figure 7(a). It is fast and creates a high quality grid. Trimmer mesh generates a volume mesh consisting of hexahedral cells and trimmed hexahedral cells at the geometry surface. The volume mesh generated for this study is shown in Figure 6(b). It is considered as optimum mesh type for simulating problems with large domain sizes as is the case in this study. A very fine mesh was used near the AUV wall and the size gradually increased to a very coarse mesh far away from the wall. The mesh was further refined at the nose and tail regions using the volumetric control option in the STAR $\mathrm{CCM}+$, in which custom mesh sizes can be defined at the desired areas.

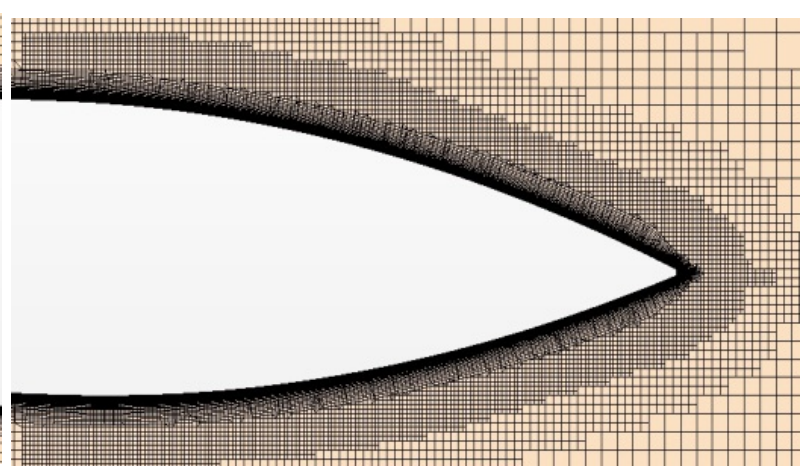

(b)

Figure 7. (a) Trimmer and Prism layer mesh at the nose region (b) Trimmer and Prism layer mesh at the tail region 


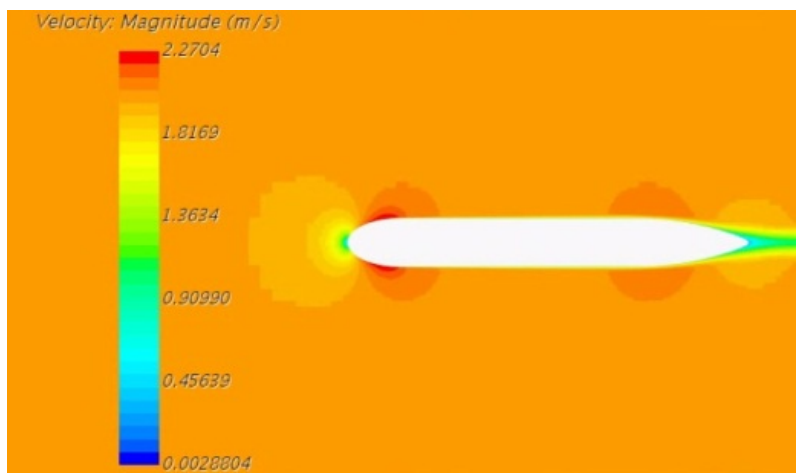

(a)

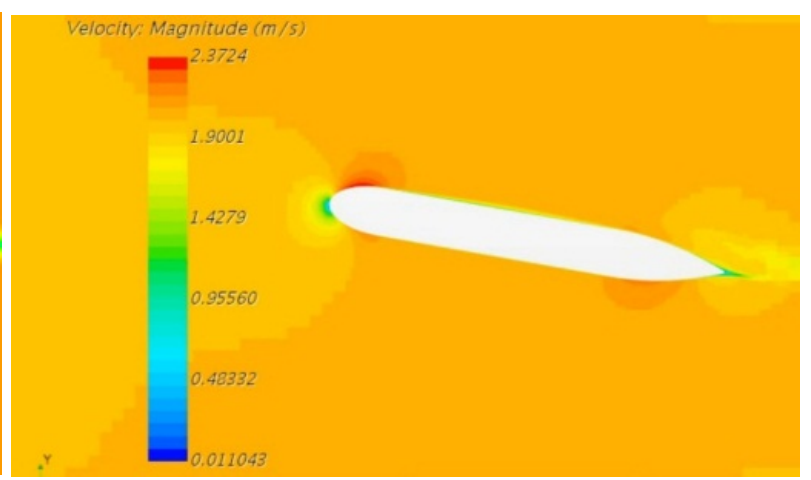

(b)

Figure 8. Velocity distribution at (a) 0 degrees (b) 10 degrees

As the boundary layer is very small and the gradients are high, a very fine mesh is to be used near the boundaries to capture the gradients correctly. Making the mesh finer overall the domain will increase the number of cells and hence the computation time. An alternative to this is to use a prism layer mesher which creates prism cells at the boundaries to capture the boundary layer phenomenon to a high level of accuracy. The mesh near the nose and tail sections with sufficient number of prism mesh can be seen in the Figure 7.

$\mathrm{k}-\epsilon$ turbulence model is the most common turbulence model used in the computational fluid dynamics for the simulation of turbulent flow characteristics. It is a two equation model and it uses two additional transport partial differential equations to solve the RANS equation. The flow conditions for all the cases of angles of attack remained the same. The walls boundaries were set to no slip condition, so that the tangential fluid velocity is set to zero at the walls and the relevant parameters like pressure and velocity are extrapolated from the adjacent cells. At the inlet and outlet face, velocity inlet $(\mathrm{v}=2 \mathrm{~m} / \mathrm{s})$ and outlet pressure boundary conditions were applied respectively.

\subsection{Results from the CFD Simulations}

CFD simulations were performed for the AUV hull of $\mathrm{L} / \mathrm{D}$ ratio 8.5 and the lift and the drag forces were estimated for angles of attack varying from 0 degrees to 20 degrees at intervals of 2 degrees. Figure 8 (a) and 8 (b) shows the velocity distribution for the hull at angles of attack 0 degrees and 20 degrees respectively. The figures visualize the stagnation point at the nose of the hull (point of maximum pressure and zero velocity), flow transition region (transition from laminar flow to turbulent flow) and flow separation region. As the angle of attack increases, these regions and points vary and this variation contribute towards the hydrodynamic loads to a great extent. At higher angles of attack, the flow gets separated way before the contracting portion of the hull, giving rise to more drag due to the formation of eddies.

\section{Comparison between the CFD and Semi-empirical Results}

The comparison between the results of the semi-empirical equations (the Hopkins equations) and the CFD simulation is made for lift force and drag force for the hull of L/D ratio 8.5. Predictions using Hopkin's equations were chosen for comparison with CFD results as they were shown to yield better results than the Allen \& Perkins and Jorgensen's formulations. The comparison between CFD and semi-empirical predictions are shown in Figure 9 along with the experimental results.

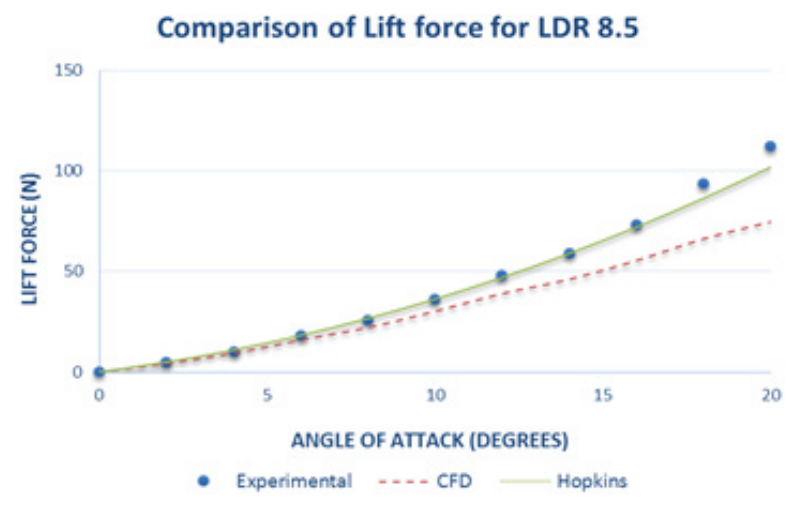

(a)

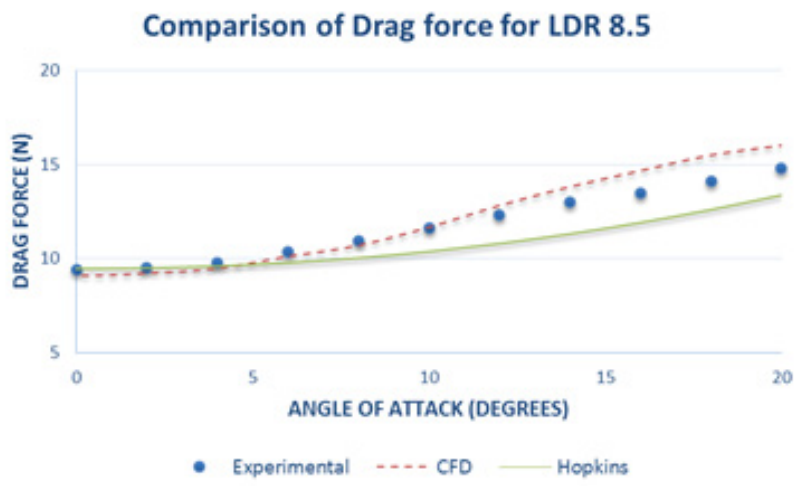

(b)

Figure 9. Comparison of results obtained from semi-empirical (using Hopkins equation) and CFD simulations with the experimental results (a) Lift force (b) Drag force 
It is observed that the lift force and the drag force predicted using semi-empirical and CFD approach match agree well with the experimental results up to an angle of attack of 10 degrees. Observing the trend, CFD is considered to be capable of capturing the lift force and drag force at higher angles of attack with a certain amount of error. The error is likely to reduce by improving the quality of the mesh and conducting a detailed mesh independence study. Since the simulations are done using the RANS equations where equations of motion are time averaged, the error is expected to be higher at higher angles of attack (above 20 degrees) where the flow around the hull is highly turbulent.

\section{Conclusions}

From the results observed and the comparisons made thereof between the experimental, semi-empirical and numerical methods in the previous section, it can be concluded that:-

1. The use of semi-empirical equations are likely to yield satisfactory results for lift force, drag force and pitching moment in cases of small angles of attack up to $10-12$ degrees only with minimum percentage error.

2. Out of the three semi-empirical equations discussed, Hopkin's equations tends to agree better than the other two for lift force and drag force up to an angle of attack of 10 degrees. However, the pitching moment is predicted reasonably up to 5 degrees only (using the Jorgensen's equation).

3. The CFD simulations can be useful for solving the cases of high angles of attack which the semi-empirical equations fail to predict accurately, such as in the comparison of drag force and pitching moment.

4. The semi-empirical equations have been validated and they can be used as a tool to calculate the hydrodynamic forces and moments on the AUV bare hull during the early stages of design of the vehicle or during the unavailability of means to study the problem experimentally or numerically.

\section{REFERENCES}

[1] Munk, M. (1924), The Aerodynamic Forces on Airship Hulls, Report No. 184, National Advisory Committee on Aeronautics (NACA)
[2] Allen, H., Perkins. E. (1951a) A Study of Effects of Viscosity on Flow Over Slender Inclined Bodies of Revolution, Report No. 1048, National Advisory Committee on Aeronautics (NACA).

[3] Allen, H., Perkins, E. (1951b), Characteristics of Flow Over Inclined Bodies of Revolution, Research Memorandum RM A50L07, National Advisory Committee on Aeronautics (NACA).

[4] Allen. H (1949), Estimation of the forces and moments acting on inclined bodies of revolution of high fineness ratio, Research Memorandum RM A9126, National Advisory Committee on Aeronautics (NACA)

[5] Hopkins. E. (1951), A Semi-empirical Method for Calculating the Pitching Moment of Bodies of Revolution at Low Mach Numbers, Research Memorandum RM A51C14, National Advisory Committee on Aeronautics (NACA).

[6] Jorgensen. L. H. (1973), Prediction of static aerodynamic characteristics for space-shuttle-like and other bodies at angles of attack from 00-1800, NASA Technical note (NASA TN D-6996)

[7] Evans J. P. (2003), Dynamic Modelling and Performance evaluation of an Autonomous Underwater Vehicle, M.E. Thesis, McGill University, Montreal, Quebec, Canada.

[8] Nahon. M. (1993), Determination of Undersea Vehicle hydrodynamic derivatives using the USAF Datcom, IEEE, pp. $283-287$

[9] Nahon, M. (1996), A Simplified Dynamics Model for Autonomous Underwater Vehicles, Autonomous Underwater Vehicle Technology AUV '96, pp. 373-379.

[10] Prestro T. (2001), Verification of a six degree of freedom simulation model for the REMUS Autonomous underwater vehicle, Master's thesis, Massachusetts institute of technology, Woods Hole Oceanographic Institution.

[11] Perrault. D. E. (2002), Autonomous Underwater Vehicles (AUV) Sensitivity of Motion Response to Geometric and Hydrodynamic Parameters and AUV Behaviours with Control Plane Faults, Doctoral Thesis, Memorial University of Newfoundland, St. John's, Newfoundland, Canada.

[12] de Barros, E. A., João L. D. Dantas, António M. Pascoal, and Elgar de Sá (2008), Investigation of Normal Force and Moment Coefficients for an AUV at Nonlinear Angle of Attack and Sideslip Range, IEEE Journal of Oceanic Engineering, vol. 33, no. 4.

[13] C. D. Williams, T. L. Curtis, J. M. Doucet, M. T. Issac and F. Azarsina, "Effects of Hull Length on the Hydrodynamic Loads on a Slender Underwater Vehicle during Manoeuvres", OCEANS 2006, Boston, MA, 2006, pp. 1-6.

[14] Jagadeesh P., Murali K., Idichandy V. D., Experimental investigation of hydrodynamic force coefficients over AUV hull form, Ocean Engineering Journal 36, 113-118. 